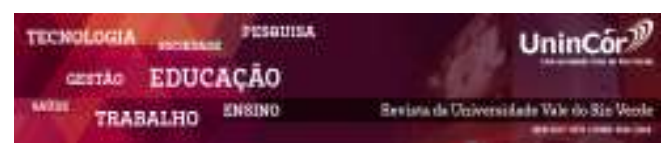

Revista da Universidade Vale do Rio Verde ISSN: 1517-0276 / EISSN: 2236-5362 Vol. 16 | n. 1 | Ano 2018

\section{A INCIDÊNCIA DE INCONTINÊNCIA URINÁRIA NOS TRATAMENTOS DE BRAQUITERAPIA E \\ PROSTATECTOMIA RADICAL NO \\ CÂNCER DE PRÓSTATA: ABORDAGEM \\ DE ABRANGÊNCIA EM PROL DA PRÁTICA CLÍNICA}

Leticia Fogaça Saud

Centro Universitário das Faculdades Associadas de Ensino leticia.fgs@hotmail.com

Gabriela Vaz Campos Centro Universitário das Faculdades Associadas de Ensino gabrielavaz_98@hotmail.com

Renan Maniezzo Centro Universitário das Faculdades Associadas de Ensino renanmaniezzo@yahoo.com.br

Yasmim Maria Pereira Marques Centro Universitário das Faculdades

Associadas de Ensino yasmimmarques29@yahoo.com.br

Laura Ferreira de Rezende Universidade de Campinas e Universidade do Estado de São Paulo laura@fae.br

\begin{abstract}
RESUMO
A escolha para o tratamento de câncer de próstata não é exclusiva. Os procedimentos são diversos e o ponto de convergência para todos é o mesmo resultado oncológico. No entanto, o que difere nesses tratamentos são os prejuízos que acometem a qualidade de vida após a terapia de escolha. Desenvolveu-se um estudo de revisão visando focar em uma sequela presente em dois tratamentos e que impacta o bem-estar do homem, a incontinência urinária. Foi feita uma comparação embasada em artigos científicos para ver a incidência da incontinência urinária após os procedimentos de prostatectomia radical e braquiterapia. O trabalho em questão foi realizado mediante a seleção e análise de alguns artigos e estudos coerentes com o tema escolhido, os quais foram encontrados nas bases de dados PubMed e Medline. A partir disso, observou-se que pacientes que se submeteram a prostatectomia radical apresentaram maiores índices de incontinência urinaria, com relação a aqueles que se trataram por braquiterapia. Os tratamentos para o câncer de próstata podem apresentar outras sequelas, como intestinais e sexuais. No momento da escolha da intervenção o paciente deve estar ciente dos impactos na sua qualidade de vida.
\end{abstract}

Palavras-chave: Braquiterapia. Prostatectomia Radical. Câncer de Próstata. Incontinência Urinária. Qualidade de Vida.

\section{INCIDENCE OF URINARY INCONTINENCE IN TREATMENTS OF BRACHYTHERAPY AND RADICAL PROSTATECTOMY IN PROSTATE CANCER: A COMPREHENSIVE APPROACH TO CLINICAL PRACTICE}


the chosen theme, which were found in PubMed and Medline databases. From this, it was observed that patients who underwent radical prostatectomy had higher rates of urinary incontinence, compared to those who underwent brachytherapy. Aiming those treatments for prostate cancer may present other sequelae, such as intestinal and sexual, at the time of choosing the intervention the patient should be aware of the impacts on their quality of life.

Keywords: Brachytherapy. Radical Prostatectomy. Prostate Cancer. Urinary Incontinence. Quality of Life.

Recebido em: 18/11/2016 - Aprovado em: 15/01/2018 - Disponibilizado em: 15/07/2018

\section{INTRODUÇÃO}

A incidência do câncer de próstata está entre 200 a 300 mil casos anualmente nos Estados Unidos e na Europa, sugerindo ser o câncer mais presente entre os homens. Sendo que, esses estão na faixa etária média de 65 anos, e geralmente são diagnosticados em suas fases iniciais (Ferrer et al,2013). Além disso, deve-se ressaltar que a segunda causa de morte por câncer entre os homens americanos é aquele que acomete a próstata, sabendo que, no ano de 2007 ocorreram 27.050 mortes nos Estados Unidos (Lin et al,2011).

Os principais tratamentos recomendados pela Associação Europeia de Urologistas são prostatectomia radial e braquiterapia (Boettcher et al,2011). Estes geralmente produzem o mesmo resultado oncológico. Contudo, os tratamentos se diferem na qualidade de vida daqueles que se submeteram a estes procedimentos (Hashine et al,2011).

A prostatectomia e a braquiterapia têm suas próprias consequências sexuais, intestinais e urinárias. A incontinência urinária é um efeito comum a ambos os tratamentos e tem grande interferência na qualidade de vida (Lin et al,2011). Portanto, o objetivo desse artigo foi desenvolver uma revisão sistemática sobre a incidência de incontinência urinária em homens submetidos aos tratamentos de braquiterapia e prostatectomia.

\section{MÉTODOS}

Foi feita uma busca de artigos nas bases de dados MEDLINE e PUBMED usando os descritores: neoplasias da próstata, braquiterapia, incontinência urinaria, prostatectomia radical e qualidade de vida em inglês. Os artigos duplicados foram eliminados. Posteriormente, excluiu-se os artigos que antecedem 2011 e os que eram de revisão sistemática. Os restantes foram rastreados e incluiu-se os artigos que mais se adequavam ao tema proposto. Figura I. 
Figura I- Fluxograma de identificação e seleção dos artigos para revisão sistemática sobre a incidência de incontinência urinária nos tratamentos de braquiterapia e prostatectomia radical no câncer de próstata

Número de relatos identificados nas bases de dados:

Pesquisador 1: 250

Pesquisador 2: 251

Índice de concordância: 0,97

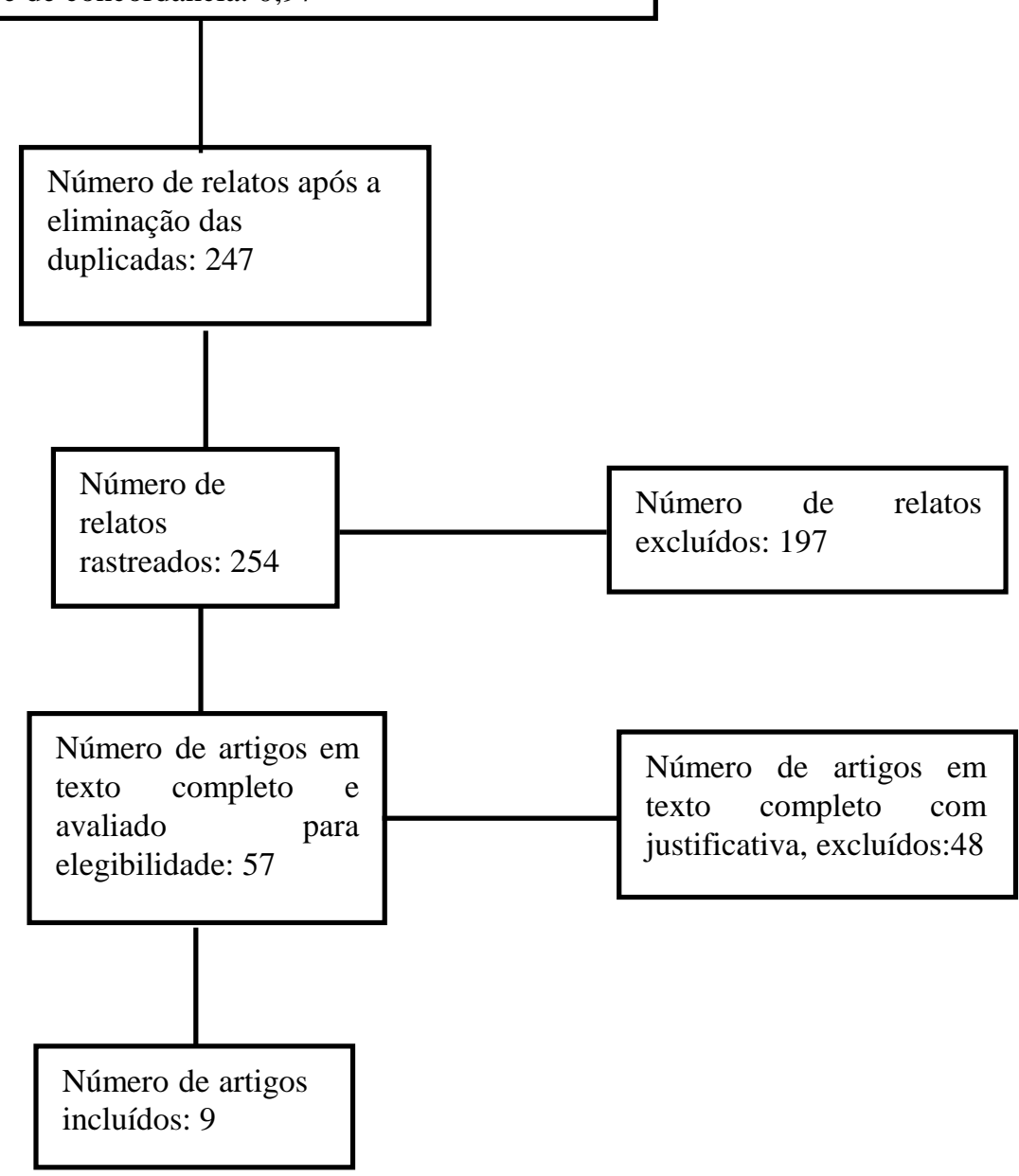

Fonte: tabela elaborada pelo próprio autor com embasamento nos dados fornecidos pelos artigos científicos.

\section{RESULTADOS E DISCUSSÃO}

$\mathrm{Na}$ tabela abaixo, encontram-se os nove artigos escolhidos. Tabela I

resultados obtidos nesses estudos a partir dos 


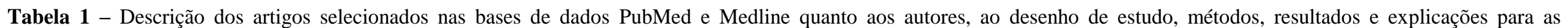
inferências.

\begin{tabular}{|c|c|c|c|c|}
\hline utor(ano) & Método & Avaliação & Resultados & Explicação dos autores \\
\hline Boettcher et al (2011) & $\begin{array}{l}\text { Estudo prospectivo longitudinal } \\
\text { com } 389 \text { pacientes. Destes, } 265 \\
\text { pacientes foi submetido a } \\
\text { prostatectomia e } 124 \text { pacientes } \\
\text { foram submetidos a } \\
\text { braquiterapia. Os homens fo- } \\
\text { ram investigados } \\
\text { individualmente com relação } \\
\text { aos sintomas miccionais antes } \\
\text { do tratamento,e } 6,12,24 \text { e } 36 \\
\text { meses após tratamento. }\end{array}$ & $\begin{array}{llr}\text { Todos os } & & \text { pacientes } \\
\text { responderam } & \text { o } & \text { questionário } \\
\text { QLQ-C30* e } & \text { o } & \text { questionário } \\
\text { ICS-male** } & \text { em r cada } \\
\text { avaliação }\end{array}$ & $\begin{array}{l}\text { Ajustado para idade e pré-tratamento dos } \\
\text { sintomas, descobriu-se que havia } \\
\text { estatisticamente mais } \\
\text { sintomas de bexiga hiperativa (OAB) } 36 \\
\text { meses após BT em comparação com os } \\
\text { pacientes tratados } \\
\text { com RP .As alterações na gravidade dos } \\
\text { sintomas da OAB ao longo do tempo ,e } \\
\text { a variabilidade dos sintomas da foram } \\
\text { significativamente maior nos pacientes } \\
\text { tratados com BT do que em pacientes } \\
\text { tratados com RP. }\end{array}$ & $\begin{array}{l}\text { A BT é significantemente associadaà } \\
\text { maiores taxas de sintomas de urgência a } \\
\text { longo prazo, mesmo após } 3 \text { anos. As } \\
\text { medidas repetidas mostram que os } \\
\text { sintomas de OAB são altamente flutuantes } \\
\text { e que nos doentes tratados com a BT, a } \\
\text { gravidade dos sintomas, bem como a } \\
\text { variabilidade deles era significativamente } \\
\text { maior do que nas aqueles pacientes } \\
\text { tratados com RP. }\end{array}$ \\
\hline Ávila et al (2015) & $\begin{array}{l}\text { Estudo observacional, } \\
\text { prospectivo de coorte com } \\
\text { acompanhamento até } 5 \text { anos } \\
\text { após o tratamento com } 580 \\
\text { pacientes. Destes } 65 \text { foram } \\
\text { tratados com prostatectomia } \\
\text { radical, } 152 \text { com a terapia de } \\
\text { radiação externa, e } 263 \text { com a } \\
\text { braquiterapia. } \\
\text {. }\end{array}$ & $\begin{array}{l}\text { Os efeitos colaterais foram } \\
\text { medidos com } \\
\text { EPIC***.M odelos Tobit } \\
\text { avaliaram o impacto do } \\
\text { tratamento e os efeitos } \\
\text { colaterais de preferência } \\
\text { pelos pacientes. }\end{array}$ & $\begin{array}{l}\text { Incontinência urinária grave apresentou } \\
\text { um impacto independente sobre as } \\
\text { preferências suscitadas ,ao passo que não } \\
\text { houve diferenças significativas entre } \\
\text { efeitos sexuais e intestinais secundários. }\end{array}$ & $\begin{array}{l}\text { Os resultados indicam que a incontinência } \\
\text { urinária é o efeito colateral com o maior } \\
\text { impacto sobre as preferências e que a } \\
\text { braquiterapia e radioterapia externa são } \\
\text { mais valorizadas do que a prostatectomia } \\
\text { radical. }\end{array}$ \\
\hline Zelefsky et al (2016) & $\begin{array}{l}\text { Estudo prospectivo longitudinal } \\
\text { com } 534 \text { pacientes. Destes } \\
\text { pacientes foram } \\
210 \text { foram cirurgia, } 171 \text { foram } \\
\text { braquiterapia, e } 153 \text { foram }\end{array}$ & $\begin{array}{l}\text { Questionários validados } \\
\text { foram utilizados no pré- } \\
\text { tratamento e aos } 3,6,9,12, \\
15,18,24,36 \text {, e } \\
48 \text { meses após . Os }\end{array}$ & $\begin{array}{l}\text { Aos } 48 \text { meses, a cirurgia teve } \\
\text { significativamente maior incontinência } \\
\text { urinária do que outros, } \\
\text { mas menos sintomas de irritação urinária } \\
\text { / obstrução. Braquiterapia e IMRT }\end{array}$ & $\begin{array}{l}\text { A seleção do tratamento deve incluir as } \\
\text { preferências do paciente e o potencial de } \\
\text { comprometimento da qualidade de vida } \\
\text { importante para o paciente. }\end{array}$ \\
\hline
\end{tabular}




\begin{tabular}{|c|c|c|c|c|}
\hline & $\begin{array}{l}\text { Radioterapia de Intensidade } \\
\text { Modulada (IMRT). }\end{array}$ & $\begin{array}{l}\text { questionários foram } \\
\text { dirigidos a função urinária, } \\
\text { incomôdo urinário,função } \\
\text { intestinal, incômodos } \\
\text { intestinais, função sexual e } \\
\text { incomôdos sexuais. }\end{array}$ & $\begin{array}{l}\text { mostraram melhor função sexual do que } \\
\text { a cirurgia. Resultados de quatro anos } \\
\text { mostraram incontinência urinária } \\
\text { persistente para a cirurgia com mais } \\
\text { sintomas urinários obstrutivos para } \\
\text { radioterapia. A função sexual foi } \\
\text { fortemente afetada em todos os grupos } \\
\text { ainda significativamente menor para } \\
\text { radioterapia. }\end{array}$ & \\
\hline Lin et al (2011) & $\begin{array}{lcr}\text { Estudo de corte transversal com } \\
64 \text { pacientes, } & \text { sendo que } 43 \\
\text { desses foram } & \text { submetidos } & \text { a } \\
\text { prostatectomia } & \text { e } 21 & \text { a } \\
\text { braquiterapia. } & & \end{array}$ & $\begin{array}{l}\text { O Estudo foi avaliado por } \\
\text { meio de um questionário } \\
\text { doméstico. }\end{array}$ & $\begin{array}{l}\text { O número de meses pós tratamento foi } \\
\text { positivamente correlacionado com a } \\
\text { função urinaria, e a idade foi } \\
\text { negativamente correlacionada com a } \\
\text { função sexual e incômodos intestinais. } \\
\text { Os resultados mostraram que pacientes } \\
\text { que receberam braquiterapia tiveram } \\
\text { melhores funções urinarias em } \\
\text { comparação a aqueles que foram } \\
\text { submetidos a prostatectomia. }\end{array}$ & $\begin{array}{l}\text { Os autores fizeram um estudo no intuito de } \\
\text { propor uma melhora na qualidade de vida } \\
\text { desses pacientes, além disso, eles } \\
\text { recomendam que antes do tratamento os } \\
\text { médicos apresentem todos as } \\
\text { consequências e benefícios de cada } \\
\text { tratamento para que o paciente decida qual } \\
\text { o melhor tratamento. }\end{array}$ \\
\hline Wallis et al (2015) & $\begin{array}{l}\text { Estudo de coorte retrospectivo, } \\
\text { com } 60476 \text { homens, sendo que } \\
14492 \text { fizeram prostatectomia e } \\
45984 \text { radioterapia. }\end{array}$ & $\begin{array}{l}\text { Foram medidos em cada } \\
\text { indivíduos complicações } \\
\text { relevantes usando bancos de } \\
\text { dados Medcare, onde foram } \\
\text { identificados códigos, a } \\
\text { partir de uma revisão cega } \\
\text { dos procedimentos de coorte } \\
\text { médicos. }\end{array}$ & $\begin{array}{l}\text { De todos os homens, } 55 \% \text { tiveram pelo } \\
\text { menos uma complicação (sexual, } \\
\text { intestinal e urinaria) que progrediu ao } \\
\text { longo de } 10 \text { anos, com pico no segundo } \\
\text { ano de tratamento. Os tratados com } \\
\text { radiação tiveram a maior taxa de } \\
\text { procedimentos urológicos e retais-anais, } \\
\text { mas uma menor taxa de cirurgia de } \\
\text { grande porte em comparação com a } \\
\text { prostatectomia. }\end{array}$ & $\begin{array}{l}\text { Os autores acharam o resultado consistente } \\
\text { com seus achados anteriores, embora a } \\
\text { magnitude dos efeitos nesse estudo tenha } \\
\text { sido menor. }\end{array}$ \\
\hline Hashine et al (2011) & $\begin{array}{l}\text { Estudo longitudinal } \\
\text { prospectivo, } 198 \text { pacientes, } 91 \\
\text { fizeram braquiterapia e } 107 \\
\text { fizeram prostatectomia }\end{array}$ & $\begin{array}{l}\text { Os pacientes que } \\
\text { concordaram em participar } \\
\text { da pesquisa relataram sobre } \\
\text { sua qualidade de vida após o } \\
\text { tratamento em que foram } \\
\text { submetidos. Foi utilizada a }\end{array}$ & $\begin{array}{l}\text { A continência urinária após a } \\
\text { prostatectomia radical retropúbica } \\
\text { melhorou ligeiramente a partir de } 12 \\
\text { meses a } 3 \text { anos, mas ainda era } \\
\text { significativamente pior depois da } \\
\text { braquiterapia. A função sexual não se }\end{array}$ & $\begin{array}{l}\text { A avaliação da qualidade de vida } \\
\text { representa uma questão importante na } \\
\text { gestão do câncer de próstata. Nossas } \\
\text { descobertas são susceptíveis de ser de } \\
\text { ajuda no desenvolvimento de um plano de } \\
\text { tratamento para pacientes com câncer de }\end{array}$ \\
\hline
\end{tabular}




\begin{tabular}{|c|c|c|c|c|}
\hline & & $\begin{array}{l}\text { International Prostate } \\
\text { Symptom Score (IPSS)****, } \\
\text { e a versão japonesa do } \\
\text { Medical Outcome Study 8- } \\
\text { itens Short Form Health } \\
\text { Survey (SF-8). Nós usamos } \\
\text { essas duas pontuações } \\
\text { resumidas para comparar o } \\
\text { RRP e grupos PPB. }\end{array}$ & $\begin{array}{l}\text { alterou entre } 12 \text { meses e } 3 \text { anos em } \\
\text { ambos os grupos. A incontinência } \\
\text { urinária em } 3 \text { anos foi correlacionada } \\
\text { com o método de tratamento e idade dos } \\
\text { pacientes, enquanto a irritação urinária / } \\
\text { obstrução foram correlacionadas com o } \\
\text { pré-tratamento. }\end{array}$ & próstata. \\
\hline $\begin{array}{l}\text { Tol-Geerdink et al } \\
\qquad(2013)\end{array}$ & $\begin{array}{l}\text { Estudo randomizado. Entre } \\
2008-2011,240 \text { pacientes com } \\
\text { câncer de próstata localizado } \\
\text { foram selecionados para ser } \\
\text { elegível para ambos a } \\
\text { prostatectomia radical (RP) e } \\
\text { radioterapia externa (RT). } \\
\text { Braquiterapia (BT) foi uma } \\
\text { terceira opção para alguns. A } \\
\text { qualidade de vida relacionada à } \\
\text { saúde foi medida pelo índice } \\
\text { compósito cancro da próstata } \\
\text { ampliada (EPIC) até } 12 \text { meses } \\
\text { após o tratamento. }\end{array}$ & $\begin{array}{l}\text { A qualidade de vida foi } \\
\text { avaliada por meio do } \\
\text { EPIC*** (Wei et al, 2000). } \\
\text { Este instrumento específico } \\
\text { da próstata inclui perguntas } \\
\text { sobre a função e sobre no } \\
\text { sistema urinário, no } \\
\text { intestino, no domínio. }\end{array}$ & $\begin{array}{l}\text { Nossos resultados sugerem que para } \\
\text { pacientes que realmente têm uma } \\
\text { escolha, os dois tratamentos, a } \\
\text { Radioterapia e a Braquiterapia } \\
\text { apresentam em média as mesmos } \\
\text { resultados de maneira positiva, e suas } \\
\text { sequelas são relativamente as mesmas. }\end{array}$ & $\begin{array}{l}\text { Mais pesquisas, com um acompanhamento } \\
\text { mais longo, precisa ser feito para confirmar } \\
\text { essa descoberta. Recomendamos que } \\
\text { futuros estudos comparando os efeitos de } \\
\text { diferentes tratamentos devem incluir } \\
\text { apenas pacientes selecionados para serem } \\
\text { elegíveis para ambos os tratamentos. }\end{array}$ \\
\hline Ferrer et al (2013) & $\begin{array}{l}\text { Estudo de } r \text { coorte } \\
\text { observacional, prospectivo, } \\
\text { com avaliação da qualidade de } \\
\text { vida, de pré-tratamento e } \\
\text { acompanhamento até cinco } \\
\text { anos após o tratamento. } 704 \\
\text { pacientes com risco baixo ou } \\
\text { intermediário de câncer de } \\
\text { próstata foram recrutados em } \\
\text { 2003-2005. }\end{array}$ & $\begin{array}{l}\text { Qualidade de vida foi } \\
\text { medida pelo questionário } \\
\text { EPIC*** com critérios de } \\
\text { urinária irritativa-obstrutiva, } \\
\text { incontinência, intestino, } \\
\text { sexual, e as pontuações } \\
\text { hormonais (que variam de 0- } \\
\text { 100). }\end{array}$ & $\begin{array}{l}\text { O impacto na qualidade de vida por } \\
\text { tratamento de braquiterapia é elevado em } \\
\text { sintomas irritativos/obstrutivos de vias } \\
\text { urinárias. Em contraponto, a } \\
\text { Prostatectomia radical aponta melhor } \\
\text { resultado na questão irritativa/obstrutiva, } \\
\text { mas foi observada uma deterioração } \\
\text { sexual nesse procedimento. }\end{array}$ & $\begin{array}{l}\text { A braquiterapia é o tratamento que causa o } \\
\text { menor impacto na qualidade de vida, } \\
\text { exceto para sintomas moderados } \\
\text { irritativos/obstrutivos de vias urinárias. O } \\
\text { estudo fornece informações valiosas a } \\
\text { longo prazo para a tomada de decisão } \\
\text { clínica, Apoiando braquiterapia como uma } \\
\text { possível alternativa para a prostatectomia } \\
\text { radical para os pacientes que procuram } \\
\text { tentativa ao tratamento curativo. }\end{array}$ \\
\hline Lorion et al (2013) & $\begin{array}{l}\text { Acompanhamento longitudinal } \\
\text { do IPSS nos cinco anos após o } \\
\text { tratamento de câncer de } \\
\text { próstata localizado: análise } \\
\text { global e tipo de tratamento }\end{array}$ & $\begin{array}{l}\text { Três grupos tratados com } \\
\text { prostatectomia radical (RP), } \\
\text { a radioterapia externa (TEN) } \\
\text { ou braquiterapia (Curie) } \\
\text { completaram o questionário }\end{array}$ & $\begin{array}{l}\text { O estudo incluiu } 40 \text { PR, } 40 \text { RT e } 40 \\
\text { Brachy. A análise por grupo de } \\
\text { tratamento, não houve diferença } \\
\text { significativa entre os grupos em três } \\
\text { meses e durante os primeiros } 4 \text { anos No }\end{array}$ & $\begin{array}{l}\text { Este estudo mostrou nenhuma degradação } \\
\text { da IPSS ou Qualidade de vida no } \\
\text { tratamento remoto de câncer de próstata } \\
\text { localizado. A incontinência urinária foi } \\
\text { parcialmente explorada. Seu estudo teria }\end{array}$ \\
\hline
\end{tabular}


IPSS e auto-Q-QV antes do tratamento, a três meses uma vez por ano durante

cinco anos. quinto ano, o grupo RT teve uma permitido uma análise melhor sobre a IPSS $* * * *$ maior do que os grupos questão urinária na vida desses pacientes. Brachy e PR 
A braquiterapia (BT) e a prostatectomia radical (RP) são tratamentos para o câncer de próstata e podem gerar efeitos adversos na função sexual, intestinal e urinaria. A incontinência urinaria é uma das principais consequências, gerando piora na qualidade de vida do paciente (Boettcher et al, 2011). Dessa forma, a escolha do tratamento deve-se basear no estilo de vida daquele que será submetido ao tratamento (Hashine et al, 2011).

Para Boettcher (2011), Ávila (2015) e Tol-Geerdink (2013), a incidência de incontinência urinaria foi maior na RP em comparação a BT. Em Boettcher (2011), a sequela foi presente em $66 \%$ dos que fizeram $\mathrm{RP}$, e em $33 \%$ dos que fizeram BT. Enquanto em Ávila (2015), 68\% dos que realizaram a RP tiveram incontinência urinaria, e apenas $34 \%$ dos que fizeram BT. Por fim, em Tol-Geerdink (2013), os que tiveram incontinência urinaria foram $55 \%$ e $12 \%$ nos que fizeram RP e BT, respectivamente.

Segundo Zelefsky (2016), após 48 meses da prostatectomia houve maiores sintomas de incontinência urinária. Em Wallis (2015) houve progressão das complicações após 2 anos da prostatectomia. Enquanto em Lorion (2013), o aparecimento das complicações surgiram após 5 anos do tratamento, igualando as complicações entre a braquiterapia e a prostatectomia nos períodos entre 3 meses a 4 anos.

O manejo do câncer de próstata é uma questão importante no impacto do bem-estar na vida do paciente (Hashine et al, 2011). Em Lin (2011), há a recomendação de que seja exposto para $\mathrm{o}$ paciente todos os benefícios $\mathrm{e}$ consequências que cada tratamento pode trazer, visando a qualidade de vida a longo prazo. Dessa maneira, segundo Ferrer (2013), o tratamento que causa menos efeito na qualidade de vida é a braquiterapia.

\section{CONCLUSÃO}

Sugere-se, a partir da revisão realizada, que a incontinência urinaria é mais incidente no tratamento de prostatectomia em relação a braquiterapia. No entanto, o tratamento para o câncer de próstata tem como consequências outras sequelas além da incontinência urinaria, como problemas intestinais e sexuais. Portanto, se mostra necessária a participação do paciente na decisão do melhor tratamento que enfrentará.

\section{REFERÊNCIAS}

ÁVILA, M.A et al. Estimating Preferences for Treatments in Patients With Localized Prostate Cancer. International Journal of Radiation

Oncology, Virgínia, Estados Unidos. v.91 n.2, p. 227 287, fev, 2015. Disponível em: <https://www.ncbi.nlm.nih.gov/pubmed/25491504>. Acesso em: 28 set. 2016.

BOETTCHER, M. et al. Overactive bladder syndrome: an underestimated long-term problem after treatment of patients with localized prostate câncer? BJU International, Oxford, Reino Unido. v.109, p. 1824-1830, jun, 2012. Disponível em:

<https://www.ncbi.nlm.nih.gov/pubmed/21952039>. Acesso em: 28 set. 2016.

FERRER, M. et al.

Quality of life impact of treatments for localized prostate cancer: Cohort study with a 5 year follow-up. Radiotherapy and Oncology, Aarhus, Dinamarca. v.108, p. 306-313, ago, 2013. Disponível em: 
<https://www.ncbi.nlm.nih.gov/pubmed/23849168>. Acessado em: 21 out. 2016.

HASHINE, K. et al. Health-related quality of life after radical retropubic prostatectomy and permanente prostate brachyterapy: A 3-year follow-up study. International Journal of Urology, Tóquio, Japão. v.18, p. 813-819, dez, 2011. Disponível em: <https://www.ncbi.nlm.nih.gov/pubmed/21995507>. Acesso em: 28 set. 2016.

LIN, Y. et al. Comparison of health-related quality of life between subjects treated with radical prostatectomy and brachyterapy. Journal of Clinical Nursing, Oxford, Reino Unido. v.21 n.13/14, p. 19061912, jul, 2012. Disponível em: <https://www.ncbi.nlm.nih.gov/pubmed/22176735>. Acesso em: 28 set. 2016.

LORION, R. et al. Suivi longitudinal de l'IPSS dans les cinq ans suivant un traitement d'un cancer de prostate localisé: analyse globale et par type de traitement. Progrès en Urologie, Paris, França. v.24, p.31-38, jan, 2014. Disponível em: $<$ http://www.sciencedirect.com/science/article/pii/S11 66708713002431>.

Acesso em: 28 set. 2016.

TOL-GEERDINK, J. et al. Quality of life after prostate câncer treatments in patients comparable at baseline. British Journal of Cancer, Londres, Reino Unido. v.108, p. 1784-1789, mai, 2013. Disponível em:

<https://www.ncbi.nlm.nih.gov/pubmed/23612450>. Acesso em: 28 set. 2016.

WALLIS, C.J.D. et al. New Rates of Interventions to Manege Complications of Modern Prostate Cancer Treatment in Older Men. European Urology, Arnhem, Holanda. v.69 n.5, p. 933-941, mai, 2016. Disponível em: <https://www.ncbi.nlm.nih.gov/pubmed/26572707>. Acesso em: 28 set. 2016.

ZELEFSKY, M.J. et al. Longitudinal assessment of quality of life after surgery, conformal brachytherapy, and intensity-modulated radiation therapy for prostate câncer. Radiotherapy and Oncology, Aarhus, Dinamarca. v.118 n.1, p. 85-91, jan, 2016. Disponível em:

<https://www.ncbi.nlm.nih.gov/pubmed/26780999>. Acesso em: 28 set. 2016.
Leticia Fogaça Saud

Discente do Curso de Medicina do Centro

Universitário das Faculdades Associadas de Ensino.

Gabriela Vaz Campos
Discente do Curso de Medicina do Centro
Universitário das Faculdades Associadas de Ensino.

\section{Renan Maniezzo}

Discente do Curso de Medicina do Centro Universitário das Faculdades Associadas de Ensino.

\begin{tabular}{l} 
Yasmim Maria Pereira Marques \\
Discente do Curso de Medicina do Centro \\
Universitário das Faculdades Associadas de Ensino. \\
\hline
\end{tabular}

\begin{tabular}{l}
\hline Laura Ferreira de Rezende \\
Mestre e Doutora pela Faculdade de Ciências \\
Médicas da Universidade de Campinas e pós-doutora \\
pela Universidade do Estado de São Paulo. \\
\hline
\end{tabular}

